
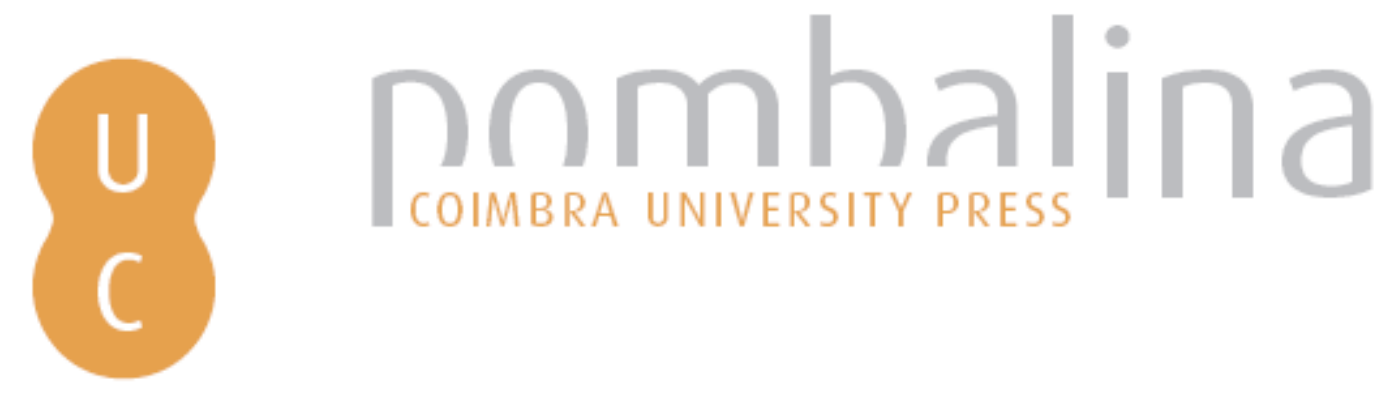

\title{
Cambios en la dinámica poblacional en el siglo V en la Cuenca de Vera: Cerro Montroy y Cabezo María
}
Autor(es):
San José, Daniel Hernández; Gutiérrez Rodríguez, Mario
Publicado por: Imprensa da Universidade de Coimbra
URL
persistente:
URI:http://hdl.handle.net/10316.2/42110
DOI:
DOI:https://doi.org/10.14195/978-989-26-1353-6_4
Accessed : $\quad$ 26-Apr-2023 03:24:07

A navegação consulta e descarregamento dos títulos inseridos nas Bibliotecas Digitais UC Digitalis, UC Pombalina e UC Impactum, pressupõem a aceitação plena e sem reservas dos Termos e Condições de Uso destas Bibliotecas Digitais, disponíveis em https://digitalis.uc.pt/pt-pt/termos.

Conforme exposto nos referidos Termos e Condições de Uso, o descarregamento de títulos de acesso restrito requer uma licença válida de autorização devendo o utilizador aceder ao(s) documento(s) a partir de um endereço de IP da instituição detentora da supramencionada licença.

Ao utilizador é apenas permitido o descarregamento para uso pessoal, pelo que o emprego do(s) título(s) descarregado(s) para outro fim, designadamente comercial, carece de autorização do respetivo autor ou editor da obra.

Na medida em que todas as obras da UC Digitalis se encontram protegidas pelo Código do Direito de Autor e Direitos Conexos e demais legislação aplicável, toda a cópia, parcial ou total, deste documento, nos casos em que é legalmente admitida, deverá conter ou fazer-se acompanhar por este aviso. 


\section{Arqueologia \\ da transição:}

entre o mundo romano

e a Idade Média

Cláudia Teixeira, André Carneiro (coords.)

IMPRENSA DA UNIVERSIDADE DE COIMBRA

ANNABLUME 


\title{
Cambios en la dinámica poblacional en el Siglo V en la Cuenca de Vera: Cerro Montroy y Cabezo María (Changes in the settlement dynamics it the Vth century in Vera basin: Cerro Monroy and Cabezo María)
}

\author{
Daniel Hernández San José (danhern@ ugr.es) \\ Universidad de Granada \\ Mario GutiérRez RodríGuez (mgrodriguez@ugr.es) \\ Universidad de Granada.
}

\begin{abstract}
Resumo - O presente estudo analisa a dinâmica populacional na bacia de Vera durante a Antiguidade Tardia, utilizando as perspectivas de análise trazidas pelos sistemas SIG. Povoados de altura, datando do século V, são analisados com particular atenção, em especial no caso de Cabezo Marías (Antas), no que poderão ser um modelo para os padrões de povoamento. Através de um estudo comparado, são analisados paralelos que permitem conhecer as elites de potentes que organizaram a área a partir do século v.
\end{abstract}

Palavras-chave - SIG, Bacia de Vera, Antiguidade Tardia, Povoamento

Aвstract - This paper analyzes the population dynamics of the Vera Basin during Late Antiquity from a territorial perspective, based on the GIS possibilities, and, more specifically, viewshed analysis. High habitats, scattered throughout the study area and dating from the 5 th century onwards, are given particular attention - especially Cabezo María (Antas), which seems to be a model for settlement patterning. Then, we conclude with a comparative study of other parallels better known that there were a group of potentes who headed the reorganization of this area in the 5 th century.

KeYwords - GIS, Vera Basin, Late Antiquity, Settlement.

La cuenca de Vera, de unos 500 $\mathrm{km}^{2}$ y situada en el Bajo Almanzora, estuvo encabezada desde la Protohistoria por el núcleo de Baria (Villaricos, Almería), de origen fenicio, constituido en municipium en época romana. Abarcando las cuencas de tres ríos (Aguas, Antas y Almanzora), el ager de Baria será objeto de una intensa explotación que no va a decaer en los siglos posteriores a los que nos ocupan. En cuanto al potencial económico y la vitalidad de su sociedad no podemos dejar de señalar el ejercicio del duovirato de uno de sus miembros (AE 1982, 632), así como la dedicatoria a mediados del siglo in por parte de la Res Publica Bariensium de un epígrafe al emperador Filipo el Árabe (SIRET 1906: 95, lám. XXIII; MENASANCH 2003:153), con una posible estatua. También destaca por su cronología tan tardía, la acción evergética de algunos miembros de su élite: es el caso de Caesianus, que construyó un templo para la ciudad (SIRET 1906: 89, 96, lám. XxIv; ANDREU 2000: 114). Queda decir que es una civitas de tercer orden 
en la escala territorial de las civitates hispanas que, por su situación, está volcada al mar, comercializando la explotación de las minas de Sierra Almagrera, los productos derivados de las actividades agropecuarias desarrolladas en su ager, actuó como nudo de comunicaciones marítimas entre Abdera (Cerro de Montecristo, Adra) y Cartagena (tal y como se desprende del Anónimo de Rávena) y encabezó la entrada al interior, hacia Basti (Cerro Cepero, Baza), a través de Tagilit (Tíjola).

En cuanto a la trayectoria histórica del poblamiento rural del territorio adscrito a Baria, las fuentes arqueológicas muestran un cambio o ruptura entre el patrón de asentamiento de este territorio desde el siglo $\mathrm{V}$, que es destacado por las investigaciones más recientes (MENASANCH 2003; CHÁVEZ 2004). E1 hecho más destacado es el traslado del núcleo originario de Baria a favor de un nuevo asentamiento, Cerro Montroy. Si hasta el siglo IV el panorama general venía marcado por una situación de eficaz explotación del territorio, con una densidad de ocupación y poblamiento del territorio equiparable a la acontecida en época del calcolítico y que no se repetiría hasta la Edad Moderna (CASTRO et al., 1996: 42), desde el siglo $V$ vemos cómo hay una serie de variaciones en la estructuración del poblamiento de la cuenca de Vera: asistimos a la reducción del número de asentamientos y a la conformación de dos grandes núcleos poblacionales, Cerro Montroy y Cabezo María. El poblamiento de la depresión de Vera, se conformaría como un hábitat disperso y con la aparición de una nueva tipología de hábitat, los asentamientos en altura. Frente al abandono de diferentes sitios arqueológicos, podemos asistir a la fundación de El Picacho de Mojácar, en el extremo sur de la depresión, Era Alta, Castillo de Mojácar, o fuera de esta cuenca, el de Los Orives. Dados los fuertes cambios que experimenta nuestra zona de estudio en el patrón de asentamiento a partir del siglo $\mathrm{V}$, desde este trabajo se propone una lectura espacial y territorial del mismo a través del análisis de una variable topográfica como la cuenca visual mediante tecnología SIG. Esto permite realizar inferencias sobre el alcance de dichos cambios de la estructura poblacional para, finalmente, hacer una propuesta del cambio social durante la Antigüedad Tardía en esta depresión.

\section{Análisis de cuencas visuales y el patrón de asentamiento}

En este trabajo se hace un acercamiento a los patrones de asentamiento y su evolución desde la óptica de la interpretación de las cuencas visuales de los sitios arqueológicos, su interrelación y su evolución en el tiempo, ya que se entiende que las relaciones visuales han jugado un papel más o menos destacado, dependiendo del momento del proceso histórico, en la estructuración de los paisajes y en las decisiones locativas de los asentamientos (WHEATLEY y GILLINGS, 2002: 201-202), llegando a formarse lo que Llobera denominó "visualscapes" o la "articulación de las propiedades visuales generadas por (o asociada a) una configuración espacial especifica" (LLOBERA, 2003: 30-31), es decir, las sociedades del pasado 
generaron pautas de territorialidad diversas que conllevaron sus propias estructuras visuales (GARCÍA et al., 2006) siendo pues la visibilidad una característica más que define los patrones de asentamiento.

De esta manera, se realizó un Modelo Digital del Terreno ${ }^{1}$ en un ambiente SIG (ArcGIS 10) ${ }^{2}$ a partir de curvas de nivel y puntos de cotas obtenidos del Instituto Geográfico $\mathrm{Nacional}^{3}$, que si bien representaba el terreno en su estado actual, se intentó restituir una variable geográfica como es la línea de costa en la Antigüedad, gracias a los datos obtenidos por el "Proyecto Costa" (ARTEAGA y HOFFMANN, 1987; HOFFMANN, 1988). Para este estudio interesan las conclusiones obtenidas para las desembocaduras de los ríos Almanzora, Antas y Aguas, donde se observó la existencia de una amplia ensenada marítima que penetraba 4 kilómetros al interior de la línea de costa actual y cuyas entradas habían sido controladas por una red de sitios arqueológicos cuya cronología oscilaba entre la época púnica y la tardoantigüedad, comenzando después, desde la Edad Media hasta la actualidad, un fuerte proceso de colmatación, provocado en gran medida por la ocupación humana, especialmente en época romana (ARTEAGA, 1995: 153; CHÁVEZ, 2004: 34), cuya tendencia ha sido regularizar la línea de costa y que ha cubierto tanto la ensenada como una serie de pequeñas penínsulas donde se ubicaban los yacimientos.

Una vez localizados los sitios se procedió a calcular la cuenca visual de cada uno de ellos en un radio de $5 \mathrm{~km}$ siguiendo las conclusiones de Zamora (2008: 127), la cual establece el radio de $5 \mathrm{~km}$ como el límite para ver sitios arqueológicos de menor entidad, tales como instalaciones agrarias o pesqueras, muy presentes en este territorio. Para determinar si la visibilidad de los sitios objeto de estudio responden a un criterio azaroso o si por el contrario ésta responde a una planificación previa relacionada con la decisión de localización de un sitio, es decir, el patrón de asentamiento, se insertaron 40 puntos aleatorios en el terreno y se calcularon sus cuencas visuales con los mismos parámetros aplicados a los sitios arqueológicos con el fin de contrastar los rangos obtenidos del cálculo del \% visible de la cuenca visual potencial $\left(78,53 \mathrm{~km}^{2}\right)$ mediante el test no paramétrico de U de Mann-Whitney. Esta técnica, ha tenido una amplia difusión en el estudio de patrones de visibilidad en sitios arqueológicos (GARCÍA et al., 2006: 185), y "se utiliza para contrastar si una diferencia entre

${ }^{1}$ La resolución de este MDE tipo TIN fue de $1 \mathrm{~m}^{2}$ por tamaño de celda así como el DEM generado a partir de éste que fue la base de los análisis de visibilidad.

${ }^{2}$ Todos los análisis y el geoprocesamiento de datos se han realizado con el mismo software (ArcGIS 10).

${ }^{3}$ Datos de la Base Cartográfica Numérica 1:25.000, Hojas 10.311, 10.312, 10.313, 10.314 y 10.321 .

4 "Investigaciones geológicas y arqueológicas sobre los cambios de la línea costera de la Andalucía mediterránea y su importancia para los asentamientos arqueológicos, especialmente fenicios, en el Sur de España". 
las medias de dos muestras es estadísticamente significativa" (GARCÍA et al., 2006: 185). En este caso, la hipótesis de trabajo es: $\mathrm{H}_{1}$ "la distribución espacial de los sitios arqueológicos está relacionada con el control visual del territorio objeto de estudio", mientras que la hipótesis nula sería: $\mathrm{H}_{0}$ "la distribución espacial de los sitios arqueológicos $\mathrm{NO}$ está relacionada con el dominio visual del territorio objeto de estudio". Como la tabla 1 refleja (Tab.1), el resultado ha validado con suficiente confianza la hipótesis de trabajo, ya que el nivel de significación está por debajo del límite establecido $(0,05 \%)$.

\begin{tabular}{|l|c|c|c|c|c|c|}
\hline & $\begin{array}{c}\text { N (sitios } \\
\text { arqueológi- } \\
\text { cos) }\end{array}$ & $\begin{array}{c}\mathrm{N} \text { (puntos } \\
\text { aleatorios) }\end{array}$ & $\begin{array}{c}\text { Suma de } \\
\text { rangos } \\
\text { (sitios } \\
\text { arqueológi- } \\
\text { cos) }\end{array}$ & $\begin{array}{c}\text { Suma de } \\
\text { rangos } \\
\text { (puntos } \\
\text { aleatorios) }\end{array}$ & $\begin{array}{c}\text { Mann- } \\
\text {-Whitney } \\
\text { U }\end{array}$ & $\begin{array}{c}\text { Nivel de } \\
\text { signifi- } \\
\text { cación }\end{array}$ \\
\hline $\begin{array}{l}\text { TOTAL } \\
\text { SITIOS }\end{array}$ & 38 & 40 & 1913,00 & 1168,00 & 348,000 & $\mathbf{0 , 0 0 0}$ \\
\hline III - V d.C. & 31 & 30 & 1221,00 & 670,00 & 205,00 & $\mathbf{0 , 0 0 0}$ \\
\hline $\begin{array}{l}\text { V-VII } \\
\text { d.C. }\end{array}$ & 19 & 20 & 487,00 & 293,00 & 83,00 & $\mathbf{0 , 0 0 3}$ \\
\hline
\end{tabular}

Tabla 1 - Resultados de U Mann-Whitney para contrastar la visibilidad de sitios arqueológicos con puntos aleatorios

De esta forma, el test U de Mann-Whitney reforzó la hipótesis de la existencia de patrones visuales, que se podían observar a nivel territorial analizando las cuencas visuales de los sitios arqueológicos a nivel cuantitativo, no sólo a nivel general sino para cada uno de los dos períodos que aquí se discuten.

Para la horquilla cronológica de los siglos III-v, los estudios del territorio de Baria coinciden en interpretar el poblamiento como un proceso tendente a la agrupación en torno a asentamientos rurales tipo villa con respecto al altoimperio (CHÁVEZ, 2004: 531-533; MENASANCH, 2003: 243), lo que estaría indicando un régimen de propiedad de la tierra tendente al latifundio, representado a nivel territorial por las grandes villae, como El Roceipón, La Torrecica, Qurénima o Cortijo Cadímar, todos ellos con una gran cuenca visual y un amplio control sobre las zonas de explotación agropecuaria, base de su existencia. Dentro de los análisis de visibilidad en época romana, las villae ocupan un puesto especial, ya que la villa se convierte en un elemento de un juego ideológico a nivel territorial, con la vista enmarca la impresión del observador con respecto al propietario y de éste con respecto al mundo exterior. Se trata pues de un símbolo del status del dominus y del poder de éste de cara al exterior (FIZ et al., 2010: 99).

En este mismo período, hay una fuerte presencia de sitios vinculados con la costa, cuya existencia vendría motivada por el control visual de la ensenada marí- 
tima así como por la carga/descarga de mercancías y el tránsito de personas. Destaca un lugar especial en el dominio visual, el sitio de Conteros II, solar donde se ubicó la civitas portuaria de Baria. Se conocen tres grandes complejos de procesado de salazón en la misma línea de costa (SIRET, 1907: 10-11). Muchos de los sitios vinculados a la bahía tienen una cronología que se remonta a la ocupación púnica y tardopúnica (Coto-1; Hoya del Pozo del Taray; Cabecicos Negros, etc.), por lo que la explotación de los recursos derivados del mar constituye una actividad continuada en el tiempo. También se localizan sitios de procesado de mineral procedente de Sierra Almagrera vinculados a la costa, así como lugares de producción cerámica, como es el caso del alfar de La Rumina o el asentamiento de Era Alta, dedicados a la exportación de manufacturas locales por vía marítima.

Finalmente, hay un grupo de sitios cuya fundación se remonta al siglo IV y que van a anteceder los cambios que se producen con mayor fuerza a inicios del siglo v d.C. Se trata de hábitats en altura como Cabezo María, La Risca o el Cortijo de la Terrera (CHÁVEZ et al., 2002: 127; CHÁVEZ, 2004: 536).

Este inicio de las reestructuraciones coincide con el abandono de las factorías de salazones de Baria (MENASANCH,2003:154) y de los yacimientos mineros de Herrerías, que como fecha temprana dejarían de funcionar con grandes ritmos de producción entre los siglos I-II d.C. siguiendo la tónica general del sureste hispano (ARBOLEDAS, 2011: 99).

Del análisis de la cuenca visual acumulada para este período, se observa un marcado interés en controlar visualmente la ensenada marítima, lugar que tuvo que protagonizar un importante papel en las relaciones socioeconómicas de la zona. Es de resaltar que Baria, representada por el sitio Conteros II $(8)^{5}$, no es de los yacimientos más percibido a pesar de que tuvo una importancia capital como municipium fiscalizador y organizador del territorio. Frente a este valor tan bajo, encontramos sitios como Cortijo de la Terrera, El Coto-1, El Coto-3 (8-10) en la ensenada marítima del Antas que tendrían valores más altos. Con respecto a los sitios de interior, representados por las villae, los valores de intervisibilidad siguen siendo bajos. Sin embargo, el Cabezo María (15) ya se configura a partir del siglo iv como un claro referente visual en el paisaje, preludiando los cambios del siglo v d.C. A nivel general, hay, como se apuntó anteriormente, un interés por el control visual de la ensenada, así como de la zona minera de Herrerías, sin embargo, el control visual del territorio, a nivel general, es bastante limitado en comparación con el período siguiente.

Por otro lado, entre los siglos v-vir destaca una reducción de los asentamientos costeros y de producción y procesado del mineral, tendencia que ya se apuntaba en el final del período anterior, permaneciendo tan sólo los sitios de El Es-

${ }^{5}$ A partir de este punto, al hablar de valores de intervisibilidad, se representa entre paréntesis la cantidad de sitios arqueológicos que guardan una relación visual directa entre sí. 
trecho, el Cerro de las Brujas, Cortijo de la Terrera y Era Alta (Tab.3), destinado éste último desde momentos altoimperiales a la transformación de recursos mineros (ARBOLEDAS, 2011: 93). Éste se trata de un fenómeno probablemente vinculado a la inseguridad e inestabilidad generalizada. Queda así reforzada la hipótesis inicial, de la existencia de estructuras visuales en el territorio fruto de la necesidad de un control visual que en este período se acrecienta por los fenómenos político sociales.

En este período, el análisis de la cuenca visual acumulada es sintomático de la situación que esta zona está experimentando. Esa selección de hábitats en altura está íntimamente relacionada con la búsqueda de una estructura visual del paisaje, ya que, con un número menor de asentamientos (19) hay un mayor control visual del territorio y del mar, origen de mercancías pero también de esas nuevas gentes que se han ido introduciendo a lo largo de dos centurias. También hay un mayor control sobre las zonas de explotación agrícola con respecto al período anterior, especialmente las circundantes a los asentamientos, así como de rutas comerciales como el inicio del pasillo de Fiñana, que desde la Prehistoria Reciente ha sido una ruta de conexión con el interior y que en época tardorromana su control toma protagonismo con la amplia cuenca visual de sitios como el Cabezo María. En cuanto a los sitios a nivel particular, siendo menos tienen unos mayores valores de intervisibilidad, como es el caso de Cabezo María (10), Cerro Montroy (10), Cerro de las Brujas (8), Almizaraque (8), Cerro de la Cueva del Murciélago (8), Castillo de Mojácar (5), El Estrecho (3), Roceipón (3), etc.

\section{LECTURA HISTÓRICA}

Por lo tanto, en el siglo $V$ se producen dos cambios destacables en la organización territorial: el traslado del espacio habitacional de Baria al muy próximo Cerro Montroy, en una posición más reducida y de fácil defensa, y la aparición de Cabezo María como el otro gran asentamiento del área entre los siglos V-VIII, rompiendo la tradición anterior donde Baria era el único centro destacado en esta zona, pero con otro aspecto que hace más interesante para su estudio. Cabezo María había tenido una ocupación marginal y secundaria en época altoimperial (CHÁVEZ 2004: 516), según se desprende de los pocos materiales de este período hallados en el asentamiento, lo que ha llevado a hablar de una auténtica "fundación" del mismo en el siglo Iv. Este fuerte contraste que hace que pase de ser un centro secundario a un núcleo de referencia en el poblamiento del territorium de Baria en cuestión de un siglo, lo que puede servirnos para comprender mejor las claves de esta transición de la Antigüedad al mundo de la Antigüedad Tardía.

En suma vamos a centrarnos a analizar la situación de la cuenca de Vera en estos momentos desde la óptica de Cabezo María, ya que Cerro Montroy sí ha sido 
objeto de una investigación más pormenorizada ${ }^{6}$. Cabe decir brevemente que Cabezo María es la denominación del yacimiento asentado sobre el cono volcánico que le da nombre, en el que predomina un tipo de roca muy característica en este ámbito local denominada verita, fruto del volcanismo lamproítico Mioceno/ Tortoniense, y que se presenta como un enclave físico inmejorable tanto por sus características defensivas como visuales. Su punto más alto es de 256 m.s.n.m., ocupando una extensión de $3 \mathrm{Ha}$. No entra dentro de la categoría estricta de poblados en altura fortificados por la ausencia de muralla: no obstante, el cono volcánico podría realizar esta misma función, ya que su acceso solo se puede hacer, con dificultad, por puntos muy concretos aún por fijar pero que pudiera ser en la cara este o sur (donde está el actual camino de acceso a la ermita que lo corona). Recientemente protegido bajo la categoría de BIC, la única excavación que en él se ha realizado fue por parte de Pedro Flores, capataz de Luis Siret (investigador muy activo en el sureste español a finales del siglo XIX y principios del xx) (SIRET 1906), en unas estructuras excavadas parcialmente en la roca del cerro y de las que realizó un croquis sobre el que recientemente se realizó su publicación (MENASANCH 2003: 180). Sobre estas estructuras hablaremos a continuación, porque también cabe recoger que Flores, a los pies del cerro, excavó unas tumbas que por la descripción que nos brinda en las memorias bien pudieran tratarse de tardorromanas debido a la presencia de un "un jarrito depositado en la parte de la cabeza" (MENASANCH, 2003: 179). Igualmente a los pies del cerro se ha identificado una villa de la que volveremos a tratar (CHÁVEZ 2000: 534).

Las estructuras excavadas por Flores serían espacios interpretados como domésticos (MENASANCH 2003: 179), y nuestro interés reside en su carácter formal: la técnica constructiva observada es la de una excavación parcial en la roca y con la presencia de una hornacina en una. Con la intención de identificar las estructuras catalogadas por Flores sobre el terreno, realizamos un reconocimiento in situ, pudiendo observar la presencia de un tipo semejante de estructuras distribuidas al menos en toda la cara norte, este y sur del cerro, que van desde la cima del mismo (actualmente ocupada por una ermita que pudiera estar levantada sobre restos de una estructura similar a las que encontró Pedro Flores a principios del xx) descendiendo hasta el mismo borde del cono volcánico, donde éste provoca un desnivel insalvable. Aparentemente, y a falta de una investigación sistemática del asentamiento, parece existir un mismo patrón constructivo en las estructuras conservadas que pudieran indicar la ocupación intencionadamente planificada en un mismo momento de este cerro, con una racionalización del espacio disponible manifestada en el aterrazamiento de éste, y que en

\footnotetext{
${ }^{6}$ Por ejemplo, desde su excavación arqueológica a la realización de una planimetría del mismo, mientras que de Cabezo María no hay ningún tipo de plano o estratigrafía, simplemente los croquis y descripciones de Flores y el estudio de sus materiales arqueológicos por prospección.
} 
apariencia satura la totalidad de su superficie del mismo. El esquema de estas casas presenta una parte excavada en roca, mientras que las estructuras exteriores se realizarían con muros de adobe o tapial sobre zócalos de mampuestos para favorecer este aterrazamiento, y su cubierta, ante la ausencia de tegulae, ha llevado a M. Menasanch a pensar en cubiertas planas también de tierra, igual que ocurría en Cerro Montroy (Menasanch 2003:179), sin embargo en la visita anteriormente mencionada se reconocieron tegulae. De hecho, y aunque con datos sustentados básicamente en materiales cerámicos de prospecciones, para esta investigadora, parece que Cabezo María reuniría un quinto de la población total de la Cuenca de Vera, dato al que habría que añadir que considera que tanto Cabezo María como Cerro Montroy absorberían casi dos tercios del poblamiento de esta depresión (MENASANCH 2003: 247) y que creemos es al menos indicativo de la trayectoria del poblamiento. Es más, creemos en la posibilidad de que ambos asentamientos hayan seguido un mismo patrón constructivo para levantar las unidades habitacionales, ya que en Montroy sabemos por la descripción de M. Menasanch que estaban construidas en buena parte contra la roca natural recortada artificialmente para dar lugar a espacios cuadrangulares: es el caso de las 'casas' 1, 3, 11, 13 y 18 de Siret (MENASANCH 2003: 158).

A ello hay que añadir las características de los materiales arqueológicos que en él aparecen muestran ciertos paralelos con los que se localizan en Cerro Montroy. Cabezo María y Cerro Montroy son los que absorben el mayor porcentaje de elementos de importación, tanto en el siglo v como el vi, mientras que los otros yacimientos que reciben material de importación son El Gitano, en el siglo v, y El Picacho, en el VI (MENASANCH 2000: 220). Para el caso concreto del Cabezo María, sus materiales cerámicos nos llevan a una cronología entre los siglos IV y VII, si bien la primera de estas centurias está poco representada, destacando la presencia de la forma 2 de TSHTM (ORFILA 2010). Por el contrario, los siglos v y vi están muy bien representados con materiales como Hayes 84, Hayes 82, Hayes 87 A, Hayes 91C, Hayes 99 y Hayes 104 para las cerámicas de vajilla, y Keay LXV y Keay LIII C para el material anfórico. Por último, hay una fuerte presencia de un material muy poco documentado en la Península Ibérica, salvo para ciertas zonas costeras, como es la Terra Sigillata Focense Tardía, también muy documentada en el Cerro Montroy, donde ya Siret documentó algunos ejemplares muy significativos (SIRET 1907) y que en el Cabezo María aparece en las formas Hayes 3B y 3D.

Finalmente, a estas características señaladas por la historiografía (la presencia de estructuras excavadas en roca, desde Flores, y la presencia de unos materiales que revelan la capacidad para atraer productos de importación), cabe añadir nuestra contribución de los resultados obtenidos a través del análisis por SIG. Que Cabezo María se constituya en un referente visual dentro del territorio de la antigua Baria y sus principales asentamientos indicaría, una vez más, la posibilidad de una planificación previa a la hora de fundarlo, en la práctica, 
como un asentamiento ex novo: tanto por las características que implican convertirlo en habitable (como comentamos, el esfuerzo para su aterrazamiento y excavación parcial de las estructuras en la roca), como por su importancia cuantitativa en número de habitantes y capacidad de consumo de materiales externos vía comercial.

Por su situación en tierras de menor calidad agrícola, en opinión de M. Menasanch el primero no sería sede de un poder institucionalizado a diferencia de Montroy (MENASANCH 2003: 254), pero en nuestra aportación queremos hacer hincapié en el carácter de Cabezo María como referente visual con relaciones de intervisibilidad desde Montroy y desde el otro asentamiento que cierra la costa de Vera, El Picacho de Mojácar, poblado en altura que aparece del siglo v al vir, es decir, con fechas similares. Por tanto, como mínimo, Cabezo María, Montroy, pero también El Picacho, muestran unas fechas similares en su aparición como referentes visuales. Por tanto Cabezo María, pese a no presentar amurallamiento, sí presenta una entidad referencial. Ante la inexistencia de una excavación no se puede barajar la presencia o no de elementos más destacados en su cima, bajo la actual ermita con origen en XVI-XVII, que pudieran dar lugar a pensar en un carácter institucional añadido. Las dificultades para dar el salto a una interpretación sobre la transición histórica en este área son numerosas y complejas ante la ausencia de una intervención arqueológica pormenorizada.

\section{UNA PROPUESTA DE TRANSICIÓN EN LA CUENCA DE VERA.}

Dicho todo esto, según los estudios realizados hasta la fecha en el siglo $\mathrm{V}$ se produce un cambio considerable especialmente en la estructura poblacional en la cuenca de Vera: se resumiría a través del declive de Baria como núcleo urbano, la aparición de Cerro de Montroy y Cabezo María como las mayores poblaciones del territorio de Vera entre los siglos v-viI, y la aparición de la tipología de asentamientos en altura (Los Orives, Era Alta, E1 Picacho...), que no implica el abandono de la totalidad de asentamientos en llano pero sí su descenso ya que el perfil que definiría el poblamiento de este momento sería el de un hábitat disperso (MENASANCH 2003: 247) y sigue la dinámica general del Mediterráneo Occidental (GUTIÉRREZ 1996: 275-276). La continuidad del poblamiento en el antiguo solar de Baria se documenta también con la existencia de una tegula hallada con una inscripción en griego $^{7}$ (ICERV 523; VIZCAÍNO, 2009: 733-735) y que pudiera incluirse dentro de un grupo de comerciantes, según la posición de L. García Moreno sobre la presencia de colonias de orientales en diferentes enclaves, siendo los más cercanos a Vera

${ }^{7}$ HENOA/ KATAK/ ITE EYTY/XH $\Sigma /$ O $\Sigma$ YEOK/ $\Sigma$ AMB/ATOY. “(Cruz) Aquí yace Eutyches, griego, hijo de Sambatius". 
los de Tortosa, Elche, Cartagena o Malaka (GARCÍA MORENO: 1972) y al hallazgo de unos nummi de época de Justiniano que también se atestiguó en la zona (VIZCAÍNO 2009: 720).

Uno de los factores tradicionalmente señalados como causa de aparición de estos asentamientos en altura es la inestabilidad política que se deriva de fuentes primarias, que desde la ruptura del limes en el 406 hacen hincapié en las acciones y movimientos de los pueblos "bárbaros" en suelo hispano. Las noticias que tenemos de acciones bélicas más próxima a Vera son las noticias que nos dan Sidonio Apolinar sobre el foedus de Honorio con los visigodos de Walia para expulsar a los vándalos y alanos de Hispania, que provocaban el caos en Calpe ${ }^{8}$ (Poemas, II 364-366), y en fechas más tardías, la destrucción que nos transmite Hidacio (Chronicon, a.460; Isidoro, Chronicon, 76) de la flota que Mayoriano estaba preparando para mandar una expedición contra los vándalos, en la cartaginense. Luego les sigue la intervención de Justiniano en el contexto de la guerra civil entre Agila y Atanagildo (Isidoro, Historiae, 46; o Jordanes, Getica, 303). Igual sucede cuando Leovigildo saquea el ager de Basti y Malaca en el c. 570 (Juan de Bíclaro, Chronicon, 12), pocos años después, y poco podemos decir de las noticias llegadas sobre las acciones bélicas entre godos y los romani milites hasta la caída de Carthago Spartaria y su destrucción a manos de Suintila C.622 (Isidoro, Historiae, 62). Pese a todo, estos momentos son ya de la primera mitad del siglo viI, y quizá se alejen de nuestro estudio, y cabe decir que la muralla de Montroy o la del coetáneo yacimiento de Los Orives (es decir, del siglo vi según MENASANCH 2003: 255) no tienen la entidad de las de Begastri o las de Tolmo de Minateda, lugares donde sí se debió dar un enfrentamiento directo. De esta forma hay una imagen de repetidas acciones bélicas, sin embargo muy repartidas en el espacio de dos siglos $(\mathrm{v}-\mathrm{VI})$ y que no nos permiten puntualizar más en base a la información literaria. La aparición de los asentamientos en altura tendría más relación con el interés por el mantenimiento del orden social y económico en un momento de crisis (GUTIÉRREZ LLORET 1996: 275ss), aunque se acepta que la necesidad de proteger un territorio ante la posibilidad, aun así algo remota para el caso de Vera, de una incursión tendría también esta función. En Vera no hay presencia de una labor de envergadura que sí se da en otros lugares: el refuerzo de un bastión en Denia, la elevación de fortificaciones como Begastri, Garres, Tolmo de Minateda, o el refuerzo de las murallas que realiza Comitiolo en el 589 en Cartagena. La mayoría de las noticias de la preparación de unas defensas realmente consistentes son del período de la década final del siglo vi hasta la caída de Cartagena, momento en que el regnum gothorum sale consolidado tras la labor de Leovigildo y

\footnotetext{
${ }^{8}$ Interpretable como Gibraltar, o como la actual Calpe alicantina, según las propias notas a la traducción castellana (editorial Gredos, 2005, nota 94 o p. 105)
} 
la superación de los cinco años de rebelión de Hermenegildo (c.579-584), claves para el posterior desarrollo del reino católico de Toledo?.

La cuenca de Vera se encontraría en un área secundaria y bien protegida geográficamente, por lo que estas acciones la afectarían relativamente. Frente a este panorama de constantes luchas, quizás exagerado por las fuentes, cabe enfrentar otro tipo de información procedente en esencia de la investigación arqueológica. De ella queremos resaltar la pervivencia de diferentes villae que en este área de Vera se considera hasta los siglos v-vII (CHÁVEZ 2004: 535). De las veinte villas de las que se tiene constancia en época altoimperial, para época bajoimperial quedarán 6 (CHAVEZ 2004: 550), que continuarán perviviendo hasta más allá del siglo v: es el caso de Roceipón, cuyos materiales revelarían una ocupación desde el Alto Imperio (CHÁVEZ 2004: 515) hasta principios del viI, siendo una villa referente en este área, con un gran programa decorativo datado en el siglo IV conocido gracias a las diferentes excavaciones de A. Pérez Casas (CHÁVEZ 2004: 21, y sobre la ocupación, CHÁVEZ 2004: 178 y MENASANCH 2003: 101). Igualmente podría suceder con otras villas que no han mostrado riqueza y ostentación de tipo propagandístico como la anterior en parte porque no han sido excavados: Cadímar (MENASANCH 2003: 69), con pervivencia hasta el siglo viI, o el gran asentamiento rural del Cortijo de los Riquelmes (MENASANCH 2003: 77, 155). La villa de Pie del Cerro María nos parece un caso a destacar por la interesante interpretación de Esther Chávez, que considera que la villa de Qurénima, con destacada importancia desde el s. I al v d.C., se abandonaría y trasladaría precisamente en este siglo $\mathrm{V}$ a los pies de Cabezo María, siendo ocupada de los siglos v al viı (CHÁVEZ 2004: 534) según la investigación del grupo Poblamiento Tardorromano y Altomedieval en la cuenca baja del río Almanzora, Almería (CAMALICH y MARTÍN 1999).

Nos parecen unos datos a resaltar, máxime si consideramos que la reorganización del territorio de Baria se realizó ya desde el siglo iII, con una reestructuración y concentración del poblamiento de época altoimperial: acabamos de citar la reducción del número de villae, pero también lo podemos ver como un proceso general (CHÁVEZ 2004: 534ss.). No se abandona el ager y su poblamiento en llano, sino que se realiza una conexión visual con los nuevos poblados en altura, tal y como E. Chávez comentó (CHÁVEZ 2004: 556-557) y que no había sido investigado específicamente hasta nuestro análisis mediante tecnología SIG.

\footnotetext{
${ }^{9}$ Este trabajo incorpora reflexiones y conclusiones del estudio realizado con la Beca de Iniciación a la Investigación 2010/11 del Plan Propio de la Universidad de Granada integrada en los contenidos del grupo de investigación HUM 178, "Paganos, Judios y Cristianos en la Antigüedad”, por Daniel Hernández San José, D., y este aspecto que señalamos podría ampliarse enla memoria de la misma: "La Iglesia y la legitimación de la violencia en la Spania visigoda: El paradigma de Hermenegildo".
} 
La pervivencia de otras villae está atestiguada en otros territorios del sureste, como sería el caso de la Vega de Granada (SÁNCHEZ et al, 2008) o el entorno murciano, donde destacan Tallante (SOLER y EGEA 2000) y también el caso de los Algezares, ésta especialmente excepcional por el hallazgo de una estructura áulica que se ha interpretado ligada a la audienta del dominus (GARCÍA BLÁNZQUEZ 2006). Otros casos en Hispania son los de Fraga (o Villa Fortunatus), también con un espacio áulico, y el de Veranés, en Asturias, reconocida como espacio de representación. Decimos que es destacable porque las dimensiones de esta estructura áulica de los Algézares es comparable a la de Veranés y mantiene unas fechas que llegan hasta bien entrado el siglo vi (GARCÍA BLÁNQUEZ 2006: 125). Por tanto, vemos la actitud de determinada aristocracia en ámbitos no tan alejados de la cuenca de Vera, donde como comentábamos había una aristocracia que desde el siglo iII da muestras de su poder, sustentada en la explotación de Sierra Almagrera pero también de la agropecuaria.

Decíamos cómo según Gutiérrez Lloret (GUTIÉRREZ 1996: 275-276), opinión que acepta E. Chávez (CHÁVEZ 2004: 549), los poblados en altura se deberían a factores de orden económico y social, en una línea de dominación de la población y su control. Si esta es una de las motivaciones principales, también creemos que el control efectivo del territorio se revela necesario tras la desaparición del poder central occidental. Podemos citar los casos de Rutilio Namaciano a principios del siglo v (De reditu suo, 20) o Sidonio Apolinar a finales del mismo siglo, como dos miembros de la más alta aristocracia que, sin embargo, acuden a sus territorios o posesiones del sur de la Galia para reorganizarlos directamente tras las invasiones germánicas y los procesos de usurpaciones imperiales (y hemos comentado antes por Sonia Gutiérrez 1996: 275-275 cómo toda la pars occidentalis asiste a unos procesos similares, como el encaramamiento de los asentamientos). Esta inestabilidad política hace que haya una necesidad de resguardar la estabilidad social, económica y política a escala local, y eso está en manos de esta aristocracia, que además actúa ejerciendo su patronazgo desde las sedes episcopales (como el propio Sidonio Apolinar acabará siendo). Un ejemplo claro es el de Mérida, donde el patronazgo de sus obispos y la representación de éstos como defensores de la comunidad romana frente a la visigoda se refleja en las Vitas Patrum Emeritensium, que encajan cronológicamente con nuestro objeto de estudio. ¿Cabría la opción de que estas fueran las medidas adoptadas por la élite de la cuenca de Vera? Es en el siglo vi cuando se eleva la muralla de mediana calidad de Montroy, y el caso de Cabezo María parece un repliegue hacia el interior, a una posición de fácil defensa tanto de amenazas procedentes del mar como del interior de país (igual que el caso de Montroy, salvaguardando el contacto comercial por mar). Esta explicación encajaría con la interpretación del traslado de la villa de Qurénima a la villa de Pies del Cerro María, como ya hemos comentado. 


\section{Conclusiones}

De este modo podemos concluir, para atestiguar o no la presencia de una aristocracia local que actúa decisivamente en el cambio del panorama general de la cuenca de Vera en el siglo v, que, primero, Montroy y Cabezo María presentan unas características semejantes en poblamiento, topografía, registro de materiales cerámicos, y algunas de sus estructuras habitacionales acentuadas en Cabezo María por su desnivel, con mayores recortes en roca, mismas techumbres, mismos materiales, y las mismas fechas. A ello se añade su carácter de ser referentes visuales en el territorio. En segundo lugar, podemos ver cómo hay una serie de villas que siguen siendo ocupadas de forma continua: algunas aristocráticas (Roceipon), otras de menor entidad (Cadímar), y podríamos ver de nuevo capacidad de actuación de esta élite si fuera cierto que trasladan la villa de Qurénima a Pie del Cerro María. De esta forma, y como tercera conclusión, vemos cómo hay una racionalización del territorio: Montroy no tiene muralla hasta siglo vi, haciéndonos pensar por el momento (a falta de otros datos) que pudiera ser más una posición de cautela que de defensa directa ante un temor inminente. Además, el alejamiento del mar por parte de los núcleos principales de población es otra característica: Hay un control efectivo del territorio que se pone en práctica y es visible en la realidad material. Por último, la administración municipal de Baria perduraría en los siglos v-vir: Baria aparece citada por última vez en las actas del Concilio de Elvira a principios del siglo iv y en el Anónimo de Rávena. Que en el siglo vi se decida amurallar Cerro Montroy en una segunda fase podría mostrarlo como referente institucional al menos en siglo vi, aún sin ir en detrimento del carácter que Cabezo María tuviera.

Dado el objeto de este estudio y la breve extensión del mismo remitimos a otro trabajo reciente y que completa los aspectos sobre el estudio de los patrones de visibilidad durante la Antigüedad Tardía en el ager bariensis (GUTIÉRREZ, HERNÁNDEZ y CHÁVEZ: 2012), pero al margen de esta matización y otras cuestiones que apoyaran la actividad de esta élite en la reorganización de la cuenca de Vera, que quedaría comprobar por excavación bien de villas, bien de Cabezo María, las conclusiones definitivas que proponemos serían: en el siglo $\mathrm{v}$ se produce una reestructuración del poblamiento de la depresión de Vera planificada y organizada, donde aparecen nuevos tipos de asentamientos (Cabezo María, Cerro Montroy, Los Orives, Era Alta,...), y el abandono de diferentes yacimientos que no implica la desaparición de otros (Roceipón, Pago de San Antón), e incluso la aparición de otros asentamientos coetáneos a Cabezo María (Nueve Oliveras, El Picacho,...), sino una selección del poblamiento. La presencia de una planificación en esta reestructuración se refuerza con los resultados del análisis SIG, que permite concluir que los asentamientos de la cuenca de 
Daniel Hernández San José

Mario Gutiérrez Rodríguez

Vera conforman una estructura visual donde Cabezo María y Cerro Montroy ocupan un papel preeminente que probablemente se relacione con su papel político, administrativo e incluso ideológico ${ }^{10}$. De hecho, la ruptura de la estructura poblacional romana se debe situar en el siglo Ix, cuando el centro administrativo de Bayra (Vera), heredero de Baria, se traslade al actual Cerro del Espíritu Santo.

10 Dado el objeto de este estudio y la breve extensión del mismo remitimos a otro trabajo reciente y más completo sobre el estudio de los patrones de visibilidad durante la Antigüedad Tardía en el agerbariensis (Gutiérrez; Hernández; Chávez 2012). 


\section{Bibliografía}

ANDREU PINTADO, J. (2000): El comportamiento munificente de las élites hispano-romanas en materia religiosa: la construcción de templos por iniciativa privada en Hispania. Iberia n. ${ }^{\circ}$ 3, p. 111-128.

ARBOLEDAS MARTÍNEZ, L. (2011): Minería y metalurgia romana en el sureste peninsular: La provincia de Almería. Sagrontom, vol. 42, p. 87-102.

ARTEAGA MATUTE, O. (1995): Paradigmas historicistas de la civilización occidental. Los fenicios en las costas mediterráneas de Andalucía. Spal, 4, p. 131-171.

ARTEAGA MATUTE, O. y HOFFMANN, G. (1987): Investigaciones geológicas y arqueológicas sobre los cambios de la línea costera en el litoral de la Andalucía mediterránea. Anuario Arqueológico de Andalucía 1986, p. 194-195.

BAÑOS SERRANO, J. (2006): El sector norte del cerro del castillo de Alhama de Murcia. Un asentamiento entre la antigüedad tardía y el mundo islámico. Antigüedad y Cristianismo XXIII, p. 81-100.

BONET PÉREZ, M. A. (1988): La economía tardorromana del SE peninsular, el ejemplo de El Puerto de Mazarrón (Murcia). Antigüedad y Cristianismo V, p. 471-502.

CAMALICH MASSIEU, M.D.; MARTÍN SOCAS, D. Eds. (1999): El territorio almeriense desde los inicios de la producción hasta fines de la Antigüedad. Un modelo: La depresión de Vera y la cuenca del río Almanzora. Sevilla.

CASTRO, P.V.; COLOMER, E., ESCORIZA, T.; FERNÁNDEZ MIRANDA, M.; FERNÁNDEZ POSSE, M.D.; GARCIA, A.; GILI, S.; GONZÁLEZ MARCÉN, P.; LÓPEZ CASTRO, J.L.; LULL, V.; MARTÍN, C.; MENASANCH, M.; MICÓ, R.; MONTÓN, S.; OLMO, L.; RIHUETE, C.; RISCH, R.; RUIZ, M.; SANAHUJA YLL, E.; TENAS, M. (1996): Territorios económicos y sociales en la Cuenca de Vera (Almería) desde c. 4000 cal ANE hasta la actualidad. In: SÁNCHEZ PICÓN, A. (Coord.) Historia y medio ambiente en el territorio almeriense, UAL, p. 35-47.

CHÁVEZ ÁLVAREZ, M.E.; CAMALICH MASSIEU, M.D.; MARTÍN SOCAS, D.; GONZÁLEZ QUINTERO, P. (2002): Protohistoria y Antigüedad en el Sureste Peninsular. El Poblamiento de la Depresión de Vera y Valle del rio Almanzora (Almería) (BAR IS 1026), Oxford.

CHÁVEZ ÁLVAREZ, M.E. (2004): Análisis del territorio durante la ocupación protobistórica y romana en la depresión de Vera y valle del rio Almanzora, 
Almería. Colección Tesis Doctorales, Servicio de Publicaciones, Universidad de La Laguna.

FIZ, I.; LÓPEZ, J.; PREVOSTI, M.; ABELA, J. (2010): In conspectupropetotiusurbis: La aplicación de diferentes métodos de análisis de visibilidad al paisaje del agerTarraconensis. Cuadernos de Prebistoria y Arqueología de la Universidad de Granada, 20, p. 97-122.

GARCÍA BLÁNQUEZ, L. A. (2006): El atrium paleocristiano de Algézares. Antigüedad y Cristianismo XXIII, p. 113-132.

GARCÍA MORENO, L.A. (1972): Colonias de comerciantes orientales en la Península Ibérica, s. V-VII. Habis, 3, p. 127-154.

(1991): El hábitat rural disperso en la Península Ibérica durante la Antigüedad Tardía (siglos V-VII). Antigüedad y Cristianismo VIII, p. 265-273.

GARCÍA SANJUÁN, L.; METCALFE-WOOD, S.; RIVERA JIMÉNEZ, T.; WHEATLEY, D.W. (2006): Análisis de pautas de visibilidad en la distribución de monumentos megalíticos de Sierra Morena Occidental. In: GRAU, I. (ed.) La aplicación de los SIG en la Arqueología del Paisaje, p. 181-200.

GONZÁLEZ BLANCO, A. (1988): La población del sureste en los siglos oscuros (IV-X). Antigüedad y Cristianismo V, p. 11-30.

GUTIÉRREZ LLORET, S. (1988): El poblamiento tardorromano en Alicante a través de los testimonios materiales. Estado de la cuestión y perspectivas. Antigüedad y Cristianismo V, p. 323-338.

GUTIÉRREZ LLORET, S., (1996): La Cora de Tudm凶r de la Antigüedad Tardía al mundo islámico. Poblamiento y cultura material, Madrid.

GUTIÉRREZ RODRÍGUEZ, M.; HERNÁNDEZ SAN JOSÉ, D.; CHÁVEZ ÁLVAREZ, E. (2012): Análisis de visibilidad e inferencias sobre el patrón de asentamiento: el Cabezo María, un caso en la depresión de Vera durante la Antigüedad Tardía. Antiqvitas, 24, p. 187-202.

LLOBERA, M. (2003): Extending GIS-based visual analysis: the concept of visualscapes. International Journal of Geographical Information Science, 17 (1), p. 25-48.

LÓPEZ MEDINA, M.J., (2004) Ciudad y territorio en el sureste peninsular durante época romana, Madrid.

MARTÍN SOCAS, D.; MEDEROS MARTÍN, A.; CHAVEZ ÁLVAREZ, M.E.; DÍAZ CANTÓN, A.; ARAMBURU ESCOLANO, E.; LÓPEZ SALMERÓN, J. (1999): Estudio del Territorio In: CAMALICH MASSIEU, M.D.; MARTÍN SOCAS, D. (eds.) El territorio almeriense desde los 
inicios de la producción hasta fines de la Antigüedad. Un modelo: La depresión de Vera y la cuenca del rio Almanzora. Sevilla.

MARTÍNEZ RODRÍGUEZ, A. (1988): Aproximación al poblamiento tardorromano en el norte del municipio de Lorca. Antigüedad y Cristianismo V, p. 543-563.

MATILLA SÉIQUER, G. (1988): El castillo de los Garres. Una fortaleza tardía en la Vega de Murcia. Antigüedad y Cristianismo V, Murcia, p. 353-402

MENASANCH DE TOBARUELA, M. (2000): Un espacio rural en territorio bizantino: análisis arqueológico de la Depresión de Vera (Almería) entre los siglos V y VII. V Reunión de Arqueología Cristiana Hispánica (Cartagena, 1998), Barcelona, p. 211-222.

MENASANCH DE TOBARUELA, M. (2003): Secuencias de cambio social en una región mediterránea. Análisis arqueológico de la depresión de Vera (Almeria) entre los siglos $V y X I$ (BAR IS 1132). Oxford.

MENASANCH DE TOBARUELA, M. (2005): Los poblados de altura: centros de los nuevos espacios sociales (Depresión de Vera, Almería. Siglos V-VIII). VI Reunión de Arqueología Cristiana Hispánica Valencia - Mayo de 2003 (Preactas), p. 87-88.

MURCIA MUÑOZ, A. J. (2000): Asentamientos rurales de los siglos V-VII d.C. En el contorno de Cartagena. V Reunió d'Arqueologia Cristiana Hispánica, p. 371-382.

ORFILA PONS, M. (2010): La vajilla Terra Sigillata Hispánica Tardía Meridional In: BERNAL, D.; RIBERA, A. (Eds.) Cerámicas hispanorromanas. Un estado de la cuestión. Cádiz.

REYNOLDS, P. (1985): Cerámica tardorromana modelada a mano de carácter local, regional y de importación en la provincia de Alicante. Lucentum, IV, p. 245-267.

REYNOLDS, P. (1995): Trade in the Western Mediterranean, AD 400-700: The ceramic evidence. (BAR IS 604) Oxford.

RUIZ MOLINA, Liborio (1988): El poblamiento romano en el área de Yecla (Murcia). Antigüedad y Cristianismo V, p. 565-598.

SÁNCHEZ LÓPEZ, E.; ORFILA PONS, M.; MORENO PÉREZ, S.(2009): Las actividades productivas de los habitantes de Florentia Iliberritana. In: ORFILA, M. (Ed.) Granada en época romana: Florentia Iliberritana. Granada.

SALVADOR VENTURA, F. (1990): Hispania meridional entre Roma y el Islam. Economía y sociedad, Granada. 
SANZ SERRANO, R. (1986): Aproximación al estudio de los ejércitos privados en Hispania durante la Antigüedad Tardía. Gerión, 4, p. 225-264.

SIRET, L. (1906): Villaricos y Herrerías. Antigüedades púnicas, romanas, visigóticas y árabes. M.R.A.H.XIV, Madrid.

SOLER HUERTAS, B., y EGEA VIVANCOS, A. (2000): El Cabildo (Tallante): la pervivencia del poblamiento en el territorium de Carthago Spartaria. $V$ Reunió d'Arqueologia Cristiana Hispánica.

UBRIC RABANEDA, P. (2003): La Iglesia y los Estados bárbaros en la Hispania del siglo V (409-507). Granada.

VALLEJO GIRVÉS, M. (1993): Bizancio y la España tardo-antigua (ss. V-VIII). Madrid.

VIZCAÍNO SÁNCHEZ, J. (2009): La presencia bizantina en Hispania (siglos VI-VII): la documentación arqueológica. Murcia.

WHEATLEY, D.W.; GILLINGS, M. (2000): Vision, perception and GIS: developing enriched approaches to the study of archaeological visibility. In: LOCK, G. (ed.): Beyond the Map. Archaeology and Spatial Technologies, NATO Science Series A, Life Sciences, vol. 321, p. 1-27.

ZAMORA, M. (2008): Territorio y espacio en la Protohistoria de la Peninsula Ibérica. Estudios de visibilidad: el caso de la cuenca del Genil, Madrid.

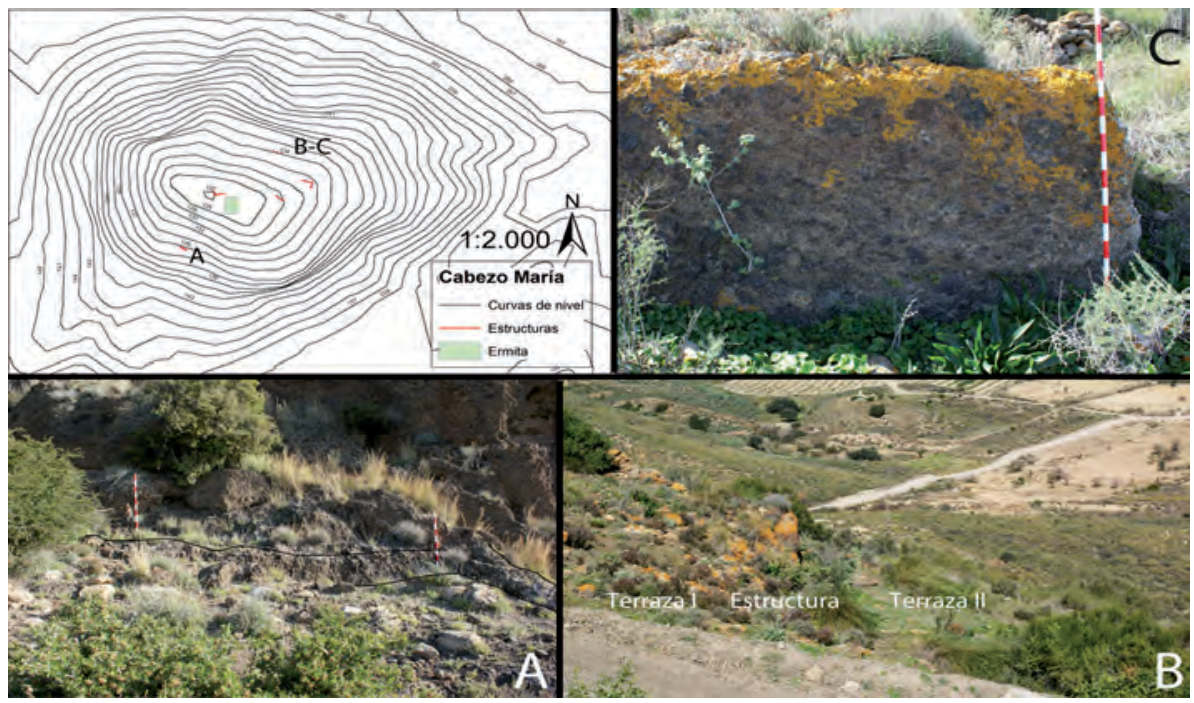

Lámina 1 - Porcentaje de la cuenca visual potencial $(78,53 \mathrm{~km} 2)$ para los siglos V-VII y III-V d.C. (en azul, hábitats en altura; en verde, instalaciones agropecuarias y villae; en naranja, zonas de procesado de mineral y artesanías pesqueras y alfareras). Los números entre paréntesis en el topónimo de los sitios arqueológicos se corresponden con su localización en la Lámina 3. 


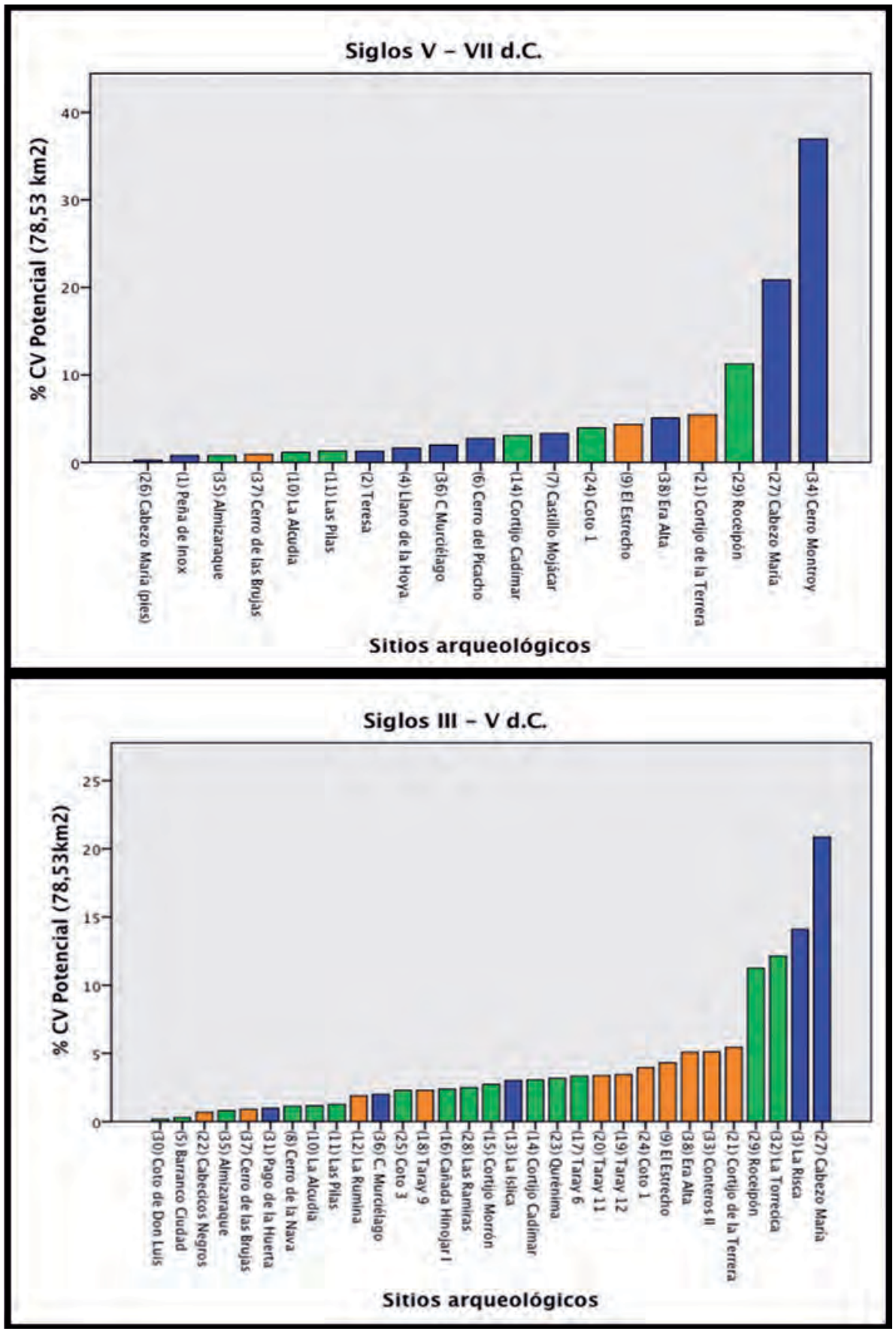

Lámina 2 - Cuenca Visual acumulada (s. III-v) y (s. v-VII) sobre Modelo Digital de Elevaciones. 
Daniel Hernández San José

Mario Gutiérrez Rodríguez

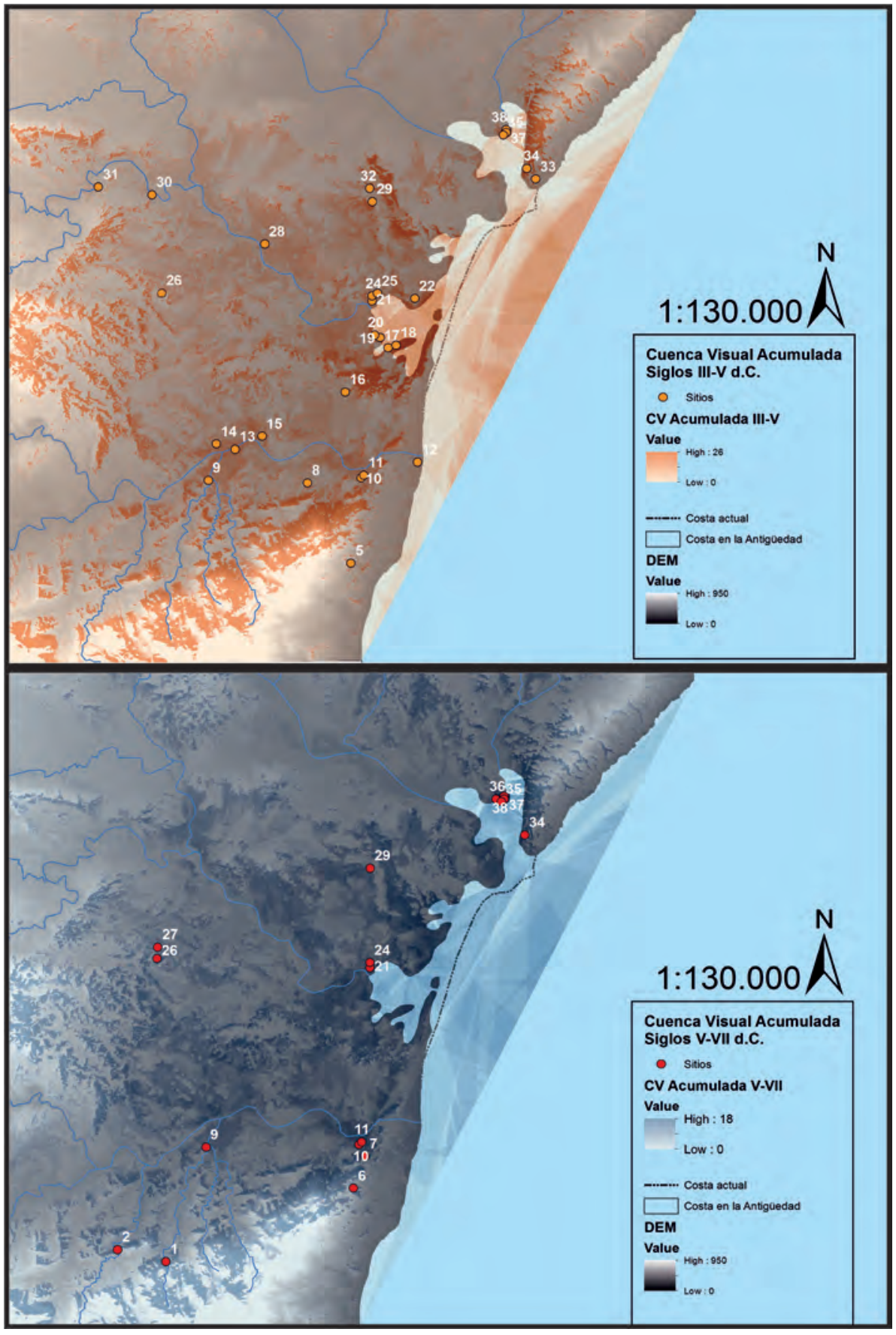

Lámina 3 - Estructuras documentadas en Cabezo María que complementan a las halladas por P. Flórez. 